Check for updates

Cite this: RSC Adv., 2018, 8, 33174

\title{
Enhanced photoresponse of a high-performance self-powered UV photodetector based on ZnO nanorods and a novel electrolyte by the piezo- phototronic effect $\uparrow$
}

\author{
Xiaoli Peng, ${ }^{a}$ Weihao Wang, ${ }^{a}$ Yiyu Zeng, ${ }^{a}$ Xinhua Pan, (D) *a Zhizhen Ye*a \\ and Yujia Zeng (DD ${ }^{\mathrm{b}}$
}

\begin{abstract}
A flexible self-powered ultraviolet (UV) photodetector based on ZnO nanorods (NRs) and a novel iodinefree quasi solid-state electrolyte was fabricated. The obtained device has a fast and high response to UV light illumination at zero bias and also shows long-term stability. The responsivity is $50.5 \mathrm{~mA} \mathrm{~W}^{-1}$ and the response time is less than $0.2 \mathrm{~s}$. Strain-induced piezo-phototronic potential within wurtzite-structured $\mathrm{ZnO}$ can optimize the performance of corresponding optoelectronic devices since it could effectively tune the charge carriers' separation and transport. The photoresponse performances of the photodetector under different upward angles (tensile strain) and downward angles (compressive strain) at $0 \mathrm{~V}$ bias were studied in detail. A $163 \%$ change of responsivity was obtained when the downward angle reached $60^{\circ}$. The enhancement could be interpreted by the piezo-phototronic effect. The piezoelectric potential (piezopotential) at the ZnO NRs/electrolyte interface can expand the built-in field, and as a result, it is easier for charge carriers to separate and transport.
\end{abstract}

Received 11th July 2018

Accepted 28th August 2018

DOI: $10.1039 / \mathrm{c} 8 \mathrm{ra05909h}$

rsc.li/rsc-advances formed. The Schottky barrier acts as the driving force to separate the generated electron-hole pairs, which makes the device operate in harsh environments without external power.

As for electrolytes, various electrolytes have been investigated. Traditionally, organic liquid electrolyte containing $\mathrm{I}^{-} / \mathrm{I}_{3}{ }^{-}$ is favored because of its high conductivity. ${ }^{14}$ While the liquid electrolyte is incline to leak out and evaporate, which results in low long-term stability and difficulty in sealing. Besides, the iodine in the electrolyte is easy to corrode the electrode and sublimate, which has a negative effect on the device. Thus, iodine-free ionic quasi solid-state electrolytes have drawn researchers' interest, since they can simultaneously hinder cohesive property of a solid and diffusive property of a liquid, and also they are environment friendly. ${ }^{15,16} \mathrm{~A}$ proper metal salt is usually added into the quasi solid-state electrolytes to improve the photoelectric performance and to coordinate with polymeric ionic liquid electrolyte to improve the solubility of solvent. ${ }^{17}$ Nevertheless, ZnO NRs/iodine-free ionic quasi solidstate electrolyte photodetector has seldom been investigated, in particular the flexible photodetector, which has great potential using in wearable devices.

In recent years, piezo-phototronic effect in $\mathrm{ZnO}$ has been utilized to enhance the performance of optoelectronic devices. ${ }^{18-23}$ Since $\mathrm{ZnO}$ is a non-central symmetric wurtzite, a piezopotential is created inside the crystal, when applying strain along the $c$-axis. Taking advantage of the strain induced piezo-phototronic effect, the charge carrier transport behavior
${ }^{a}$ State Key Laboratory of Silicon Materials, Cyrus Tang Center for Sensor Materials and Applications, School of Materials Science and Engineering, Zhejiang University, Hangzhou 310027, People's Republic of China. E-mail: panxinhua@zju.edu.cn; yezz@zju.edu.cn; Fax: +86571 87952124; Tel: + 8657187952187

${ }^{b}$ Shenzhen Key Laboratory of Laser Engineering, College of Optoelectronic Engineering, Shenzhen University, Shenzhen 518060, People's Republic of China

$\dagger$ Electronic supplementary information (ESI) available. See DOI: 10.1039/c8ra05909h 
across a Schottky junction could be effectively tuned. Zhang et al. reported a UV photodetector which shows an enhanced UV sensing capability on externally applying tensile strains, unfortunately, the UV sensing performance was still poor; the responsivity was only $0.8 \mathrm{~mA} \mathrm{~W}^{-1}$ at $0 \mathrm{~V}$ on a $0.753 \%$ tensile strain. ${ }^{11}$

In this work, a flexible self-powered UV photodetector was successfully fabricated based on the ZnO NRs and an iodinefree quasi solid-state electrolyte containing potassium iodide (KI), polyethylene oxide (PEO) and 1-methyl-3propylimidazolium iodide (PMII). The photodetector shows large photocurrent, fast photoresponse speed and high responsivity at zero bias. Besides, the photoresponse performances of the photodetector under different upward angles (tensile strains) and downward angles (compressive strains) at $0 \mathrm{~V}$ bias were studied in detail. We used the energy band diagrams of the device with and without strain to interpret the strain-modulated mechanism.

\section{Experimental}

\section{Synthesize ZnO NRs on polyethylene terephthalate (PET) substrate}

All the chemicals in this work were of analytical grade and used directly without any further purification. Commercial flexible indium-doped tin oxide (ITO) coated PET (sheet resistance is 6 ohm sq ${ }^{-1}$ and the thickness is $125 \mu \mathrm{m}$ ) was used as the substrate. The flexible substrate was cleaned with acetone, ethanol and deionized water in sequence each for 30 minutes. Before the deposition process, the flexible substrate was cleaned by $\mathrm{N}_{2}$ and part of the substrate was covered by the Kapton tape to serve as the electrode. A ZnO seed layer was deposited on the ITO coated PET substrate by pulsed laser deposition (PLD) for 10-15 minutes at room temperature. The oxygen pressure and energy of the laser are $1 \mathrm{~Pa}$ and $300 \mathrm{~mJ}$, respectively. Then, $\mathrm{ZnO}$ NRs were prepared by a simple hydrothermal method. ${ }^{24-26}$ The coated substrate with the coating side upside-down was immersed in a growth solution including $20 \mathrm{mM}$ zinc nitrate and $20 \mathrm{mM}$ hexamethylenetetramine $\left(\mathrm{C}_{6} \mathrm{H}_{12} \mathrm{~N}_{4}, \mathrm{HMT}\right)$ loaded in a Teflon liner stainless-steel autoclave at $85{ }^{\circ} \mathrm{C}$ for $4-5 \mathrm{~h}$. After the reaction was complete, the as-grown $\mathrm{ZnO}$ NRs were thoroughly rinsed with ethanol and deionized water carefully followed by a drying step in an oven at $60{ }^{\circ} \mathrm{C}$ overnight.

\section{Preparation of quasi solid-state electrolytes}

The iodine-free quasi solid-state electrolyte was prepared by dissolving $50 \mathrm{mg}$ of PMII in $2.5 \mathrm{ml}$ acetonitrile, and stirred for about $30 \mathrm{~min} .0 .03 \mathrm{~g}$ KI was added into the above solution under stirring. Afterwards, $0.2 \mathrm{~g}$ PEO was added and stirred overnight to obtain the desired homogeneous electrolyte. The electrolyte solutions were kept under stirring for about $1 \mathrm{~h}$ before each measurement to assure the electrolyte homogeneous. ${ }^{17}$

\section{Device fabrication and characterization}

$30 \mu \mathrm{l}$ of the PMII-based quasi solid-state electrolyte was dropped onto the as-grown $\mathrm{ZnO} \mathrm{NRs}$ and spun at a rate of $3000 \mathrm{rpm}$ for
$30 \mathrm{~s}$, repeating three times. A $20 \mathrm{~nm}$ thick Pt film was then deposited on the ITO-PET substrate by electron beam evaporation, serving as the counter electrode. A PET-based sealant material was used to bind the two electrodes together. The active area of the UV device was $1 \times 1 \mathrm{~cm}^{2}$. The schematic diagram of the device structure is presented in Fig. S1 in the ESI. $\dagger$ All the measuring procedures are under the same testing condition. The morphology and structure of the samples were characterized by a field emission scanning electron microscopy (FE-SEM Hitachi S-4800). The temporal response of the UV detector was measured by illuminating the devices with a UVALED. The UV-vis absorption spectra were recorded with a spectrophotometer (UV-3600). The current-voltage (I-V) characteristics were measured using an Agilent E5270B parameter analyzer under ambient conditions.

\section{Results and discussion}

Fig. 1(a) shows the SEM image of ZnO seed layer on ITO coated PET substrate. ZnO seed particles are uniform and dense, which is beneficial for obtaining uniform ZnO NRs. The typical topview SEM image and cross-sectional view of ZnO NRs are shown in Fig. 1(b) and (c), respectively. The ZnO NRs show typical hexagonal morphology and grow epitaxially on the PET substrate with preferred $c$-orientation due to the existence of the pre-deposition of the $\mathrm{ZnO}$ seed layer. The $\mathrm{ZnO}$ NRs exhibit vertical and uniform with an average length of $\sim 0.81 \mu \mathrm{m}$ and a diameter of $\sim 60$ to $130 \mathrm{~nm}$. It can be seen that gaps are well distributed between the ZnO NRs, which facilitate the penetration of the electrolyte. The UV-vis absorbance spectrum of ZnO NR is shown in Fig. 1(d), which clearly shows that it has a significant absorption below a wavelength of $400 \mathrm{~nm}$. As for a direct band gap material, the absorption coefficient $\alpha$ as a function of photon energy $h v$ can be determined as:

$$
(\alpha h v)^{2}=A\left(h v-E_{\mathrm{g}}\right)
$$
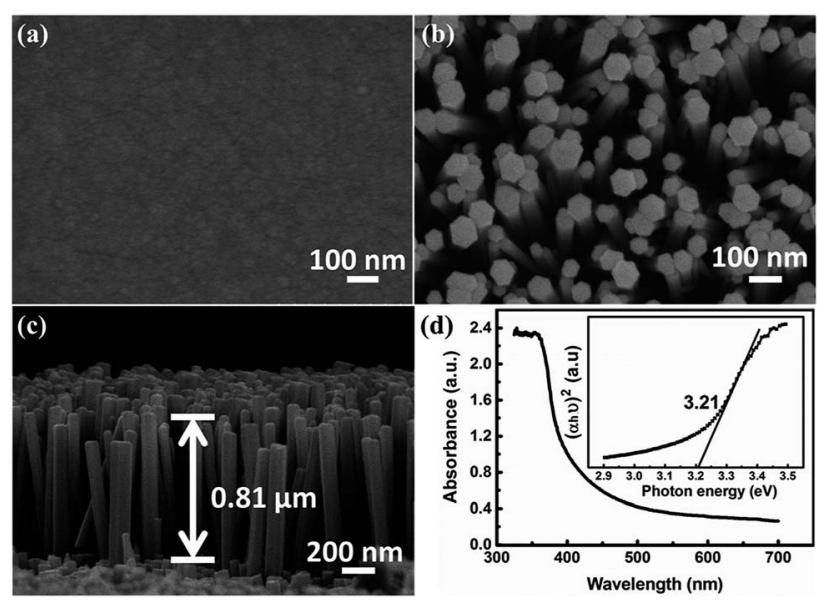

Fig. 1 (a) SEM image of ZnO seed layer on ITO coated PET substrate, (b) the top-view SEM image of ZnO NRs, (c) the cross-sectional view of $\mathrm{ZnO} N R s$, (d) the UV-vis absorbance spectrum of ZnO NRs. The inset is the corresponding band gap values derived from extrapolating the graph of $(\alpha h v)^{2}$ vs. $\left(h v-E_{\mathrm{g}}\right)$. 
Here, $A$ is constant and $E_{\mathrm{g}}$ is the band gap energy. We plotted the curve of $(\alpha h v)^{2} v s$. (hv-E $\left.E_{\mathrm{g}}\right)$, as shown in the inset of Fig. 1(d). From the linear extrapolation of the curve, the bandgap of $\mathrm{ZnO}$ NRs can be determined to be about $3.21 \mathrm{eV}$.

The XRD pattern of ZnO NRs is presented in Fig. S2 in the ESI. $\dagger$ In addition to the substrate diffraction peaks, there is a sharp diffraction peak at $34.4^{\circ}$, corresponding to the (002) crystal plane of wurtzite ZnO phase (JCPDS No. 36-1451), indicating that ZnO NRs have a good $c$-axis preferred orientation, which further confirms the results of SEM.

To further investigate the photoelectric properties of the UV detector, the $I-V$ characteristics curves both in dark conditions and the illumination of UV light with a power density of $60 \mu \mathrm{W}$ $\mathrm{cm}^{-2}(\lambda=365 \mathrm{~nm})$ are shown in Fig. 2(a). $I-V$ curves of the device both in darkness and illumination display a Schottky barrier like behavior, reflecting that the heterojunction structure has been successfully obtained. At $0 \mathrm{~V}$, there is an obvious difference between dark current and photocurrent, indicating its self-powered mode of operation. The Schottky barrier acts as the driving force to separate the generated electron-hole pairs. The responsivity $(R)$ is used to indicate the photocurrent efficiency. It is determined as:

$$
R=\frac{I_{\text {photo }}}{P A}
$$

Here, $I_{\text {photo }}$ represents the photocurrent, $P$ is the optical signal power density and $A$ is the active area of the UV device. The photocurrent is $3.03 \times 10^{-6} \mathrm{~A}$, which is much larger than dark current. In this work, the responsivity is $50.5 \mathrm{~mA} \mathrm{~W}^{-1}$, which is considerably higher than the value recently reported by other groups. ${ }^{27-31}$ Since the electrolyte contains $0.03 \mathrm{~g}$ KI and $0.2 \mathrm{~g}$ $\mathrm{PEO}$, in this case, there is an amorphous matrix for $\mathrm{K}^{+}$fully (a)

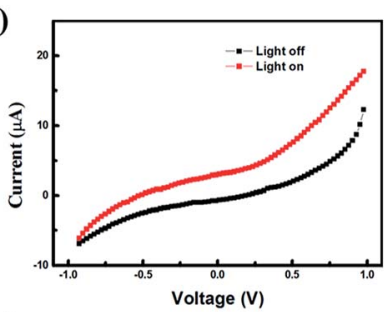

(c)

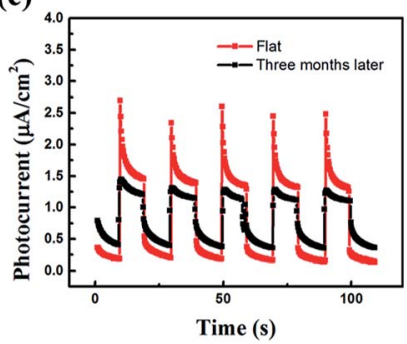

(b)

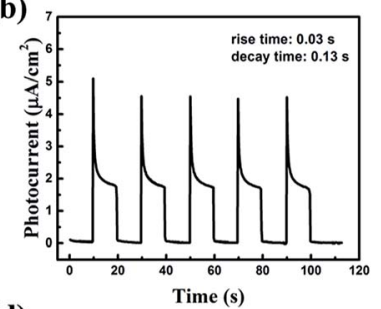

(d)

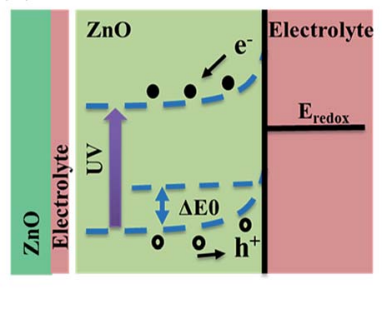

Fig. 2 (a) $I-V$ characteristics of the as-fabricated UV detector under dark conditions and the illumination of UV light. The power of the UV light used in all the experiments is $60 \mu \mathrm{W} \mathrm{cm} \mathrm{cm}^{-2}(\lambda=365 \mathrm{~nm})$. (b) The photocurrent response of the device at the zero bias. Five repeated cycles of switching UV light on (10 s) and off (10 s) were recorded. (c) Photocurrent response of a UV detector three months later. (d) Energy band bending of the device and the self-powered sensing mechanism at the zero bias. coordinating with PEO, which would facilitate the mobility of charge carrier and interfacial contact between ZnO NRs and electrolytes. ${ }^{17}$ As a result, the free charge carrier amount increases and the transfer of the charge carrier is promoted.

Fig. 2(b) shows photocurrent response of the UV detector, which was measured at $0 \mathrm{~V}$ under an intermittent irradiation of $365 \mathrm{~nm}$ UV light. Five repeated cycles of switching the $60 \mu \mathrm{W}$ $\mathrm{cm}^{-2}$ UV light on (10 s) and off (10 s) were recorded. Interestingly, there are two stages in each cycle of the curves. In the first stage, a sharp current peak emerged as soon as the UV light turned on. In the second stage, the photocurrent decreased quickly at first and then reached a steady plateau until turned off the light. We have demonstrated that this phenomenon had little to do with the stability of UV light (the photoresponse of covering sample and uncovering sample are presented in Fig. S3 in the ESI $\dagger$ ). We designed a comparative experiment including two groups to illustrate this. For group 1, we just switched the light on (10 s) and off (10 s) and recorded the photocurrent response. For group 2, at first, we turned on the light and at the same time used a black board to block the light until the light was stable. Then we removed the black board and recorded the photocurrent response. We could see that there was no difference between the two curves, reflecting that this "two-stages phenomenon" might mainly origin from the material. From a previous report, owing to the non-central symmetric crystal structures of wurtzite $\mathrm{ZnO}$, temperature changes will induce pyroelectric potentials across $\mathrm{ZnO} .^{32,33}$ The sharp peaks might stem from the combination of photovoltaic and pyroelectric effect under the UV light. While in the second stage, the photocurrent reached steady because the pyroelectric potential disappeared as the temperature stayed constant. Noting that the photocurrent of the first and the second stage are $4.55 \times$ $10^{-6} \mathrm{~A} \mathrm{~cm}^{-2}$ and $2.05 \times 10^{-6} \mathrm{~A} \mathrm{~cm}^{-2}$, respectively, indicating $220 \%$ increasing on photocurrent by pyroelectric effect. In each cycle, the photocurrent had no distinct degradation and the response process was fast. In fact, the response time is a critical parameter for the practical application of a UV photodetector. Correspondingly, the rise and decay time are 0.03 and $0.13 \mathrm{~s}$, respectively, which is shorter than the values published previously, indicating a rapid photo response behavior at $0 \mathrm{~V}$ bias. $^{34,35}$ To illustrate the stability of the device, Fig. 2(c) depicts photoresponse curves of a sample and the same sample three months later. In each cycle, there is no apparent decay on photoresponse. The rise and decay time are $0.04 \mathrm{~s}$ and $0.14 \mathrm{~s}$, respectively. Three months later, the rise and decay time are $0.34 \mathrm{~s}$ and $0.60 \mathrm{~s}$, which suggest no distinct difference, indicating good time stability. Nevertheless, the photocurrent is $\sim 1.25 \times 10^{-6} \mathrm{~A} \mathrm{~cm}^{-2}$, which is slightly smaller than the value $\left(\sim 1.55 \times 10^{-6} \mathrm{~A} \mathrm{~cm}^{-2}\right)$ three months ago. This is because part of the solvent has volatilized inevitably, resulting in the decrease of charge carrier in the electrolyte. In the electrolyte, PEO is used to solidify the liquid electrolyte to obtain the quasi solidstate electrolyte. Thus, the electrolyte is hard to volatilize and easy to be sealed. Anyhow, the quasi solid-state electrolyte is comparably stable. Compared with liquid electrolyte, it is easier to seal and harder to leak out. It should be noted that pyroelectric effect measured three months later is negligible 
compared with that measured three months ago. This phenomenon attributes to the environment temperature, since the environment temperature has a great impact on pyroelectric effect, the higher the temperature, the lower the pyroelectric effect.

The potential mechanism of UV photodetector at $0 \mathrm{~V}$ is shown in the Fig. 2(d). The Fermi level $\left(E_{\mathrm{f}}\right)$ of $\mathrm{ZnO}$ is higher than the redox potential of the electrolyte $\left(E_{\text {redox }}\right)$. When ZnO NRs contact with electrolyte in the dark, electrons diffuse from $\mathrm{ZnO}$ NRs to electrolyte and holes diffuse in the opposite direction. As a result, a built-in electric field orienting from ZnO NRs to electrolyte is formed, and the band bending at the interface between ZnO NRs and the electrolyte. When the UV light (365 $\mathrm{nm}$ ) illuminates on the device, electron is excited from valence band $(\mathrm{VB})$ to conduction band ( $\mathrm{CB}$ ), leaving behind a hole. Subsequently, the generated electron-hole pairs are forced to separate by the built-in electric field. On one hand, the holes migrate to the $\mathrm{ZnO}$ NRs/electrolyte interface and get captured by the $\mathrm{I}^{-}$in the electrolyte $\left(\mathrm{h}^{+}+\mathrm{I}^{-} \rightarrow \mathrm{I}_{3}{ }^{-}\right)$. On the other hand, the electrons travel along the ZnO NRs to FTO, and then transfer to the Pt layer of counter electrode through the external circuit. The $\mathrm{I}_{3}{ }^{-}$is reduced to $\mathrm{I}^{-}$at the counter electrode by the electrons $\left(\mathrm{I}_{3}{ }^{-}+\mathrm{e}^{-} \rightarrow \mathrm{I}^{-}\right)$. In this process, the circuit can keep running without any external bias. Besides, compared with the traditional electrolyte, the device can work smoothly without $\mathrm{I}_{2}$ avoiding the corrosiveness and sublimate of $\mathrm{I}_{2}$. What's more, the photocurrent can be generated repeatedly (due to the photovoltaic effect).

As has been reported in previous works, piezo-phototronic polarization at the interface could tune the band structure and change transport properties without changing the interface structure. ${ }^{36-38}$ Here, the strain effect on the UV photodetector was further investigated. By applying different bending curvatures, the strain can be calculated as ${ }^{39}$

$$
\varepsilon= \pm \frac{D}{2 R}
$$

where $D$ is the thickness of the substrate which is constant and $R$ is the local radius. In this work, we use curving angles to represent the strain because there is a positive correlativity between the strain and the curving angle. With the increasing of the curving angles, the local radius will decrease. As a result, the strain increases with the increasing of angles.

The photocurrent response curves of the device under varying downward angles (compressive strains) at zero bias are shown in Fig. 3(a). The photocurrent increases step by step with the increasing of the downward angles, namely, the compressive strains. To further investigate the strain modulation on the performance of the UV photodetector, we plotted the curves of the responsivity versus angles in Fig. 3(b). The responsivity increases monotonically with the increase of the curving angles, and about $63 \%$ enhanced responsivity can be got when the curving angles up to $60^{\circ}$. The response time is another key parameter for a UV photodetector. We can see from Fig. 3(a) that there is no obvious decay in response time when increasing the angles. That is, increasing the angles can increase the responsivity of the device owing to the piezo-phototronic effect,
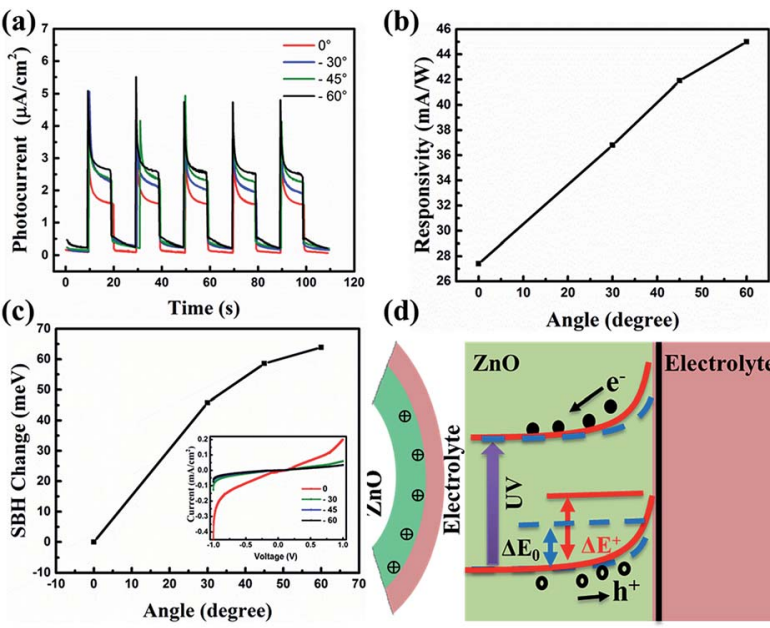

(d)

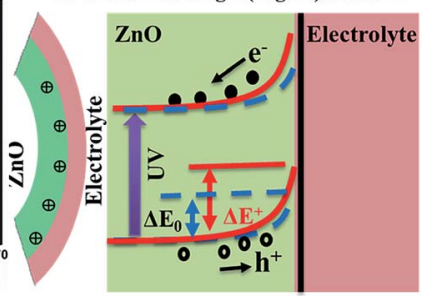

Fig. 3 (a) Photocurrent response curves of the device under varying angles (compressive strains) at zero bias. (b) The responsivity as a function of angles. (c) Derived SBH change with variable angles; (inset) $I-V$ characteristics under different angles. (d) Schematic energy band structure of the device with and without the presence of a negative piezopotential, as shown in solid red and dotted blue lines, respectively.

while does not harm the response time. To reveal the mechanism of the strain-enhanced performance of the device, we test the $I-V$ characteristics of the device under different angles, as shown in Fig. 3(c) (inset), exhibiting Schottky barrier behavior. The threshold voltage increases with the increased angles. We also calculated the change of Schottky barrier height (SBH) through thermionic emission model. According to the thermionic emission model, the reverse saturation current $I_{0}$ can be expressed $^{\mathbf{4 0}}$

$$
I_{0}=A A^{*} T^{2} \exp \left(\frac{-q \Phi_{\mathrm{b}}}{K T}\right)
$$

where $A$ is the contact area, $A^{*}$ is the effective Richardson constant, and $\Phi_{\mathrm{b}}$ is the junction barrier height. Assuming that the contact area $A$, effective Richardson constant $A^{*}$, and impurity density are independent of curving angle, the change of barrier height $\Delta \Phi_{\mathrm{b}}$ can be derived as

$$
\text { In } \frac{I_{\varepsilon}}{I_{0}}=-q \Delta \Phi_{\mathrm{b}} / K T
$$

where $I_{\varepsilon}$ and $I_{0}$ are the currents measured at fixed bias $(0 \mathrm{~V})$ with and without strain.

The SHB change with variable angles is plotted in Fig. 3(c), revealing that the SHB change increases with the increased angles, which promotes the separation and extraction of the photo generated electro-hole pairs and thus increases the photocurrent of the device. The schematic energy band structure of the device with and without the presence of a negative piezopotential is shown in Fig. 3(d). Owing to the preferred $+c$ orientation growth of $\mathrm{ZnO} \mathrm{NRs}$, a negative piezopotential will be induced at the interface, repelling electrons away from the interface and consequently further depleting the interface and raising the barrier height from $\Delta E_{0}$ to $\Delta E^{+}$. The increased barrier height broadens the depletion region and strengthens the built 
(a)

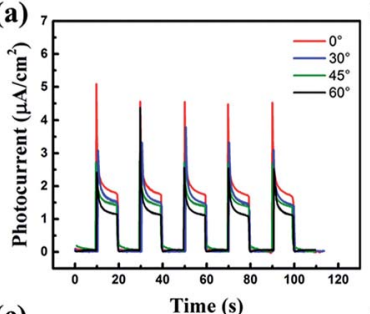

(c)

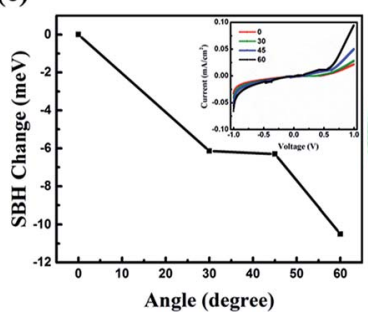

(b)

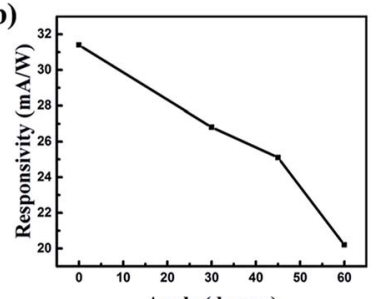

(d)

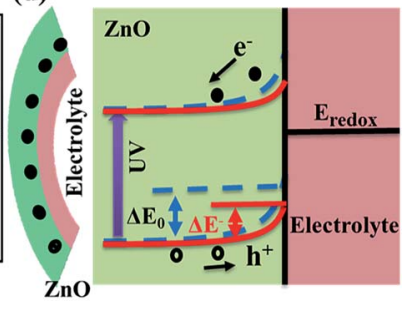

Fig. 4 (a) Photocurrent response curves of the device under varying angles (tensile strains) at zero bias. (b) The responsivity as a function of angles. (c) Derived SBH change with variable angles; (inset) I-V characteristics under different angles. (d) Schematic energy band structure of the device with and without the presence of a positive piezopotential, as shown in solid red and dotted blue lines, respectively.

in electric field. As a result, more carriers can be separated more effectively, leading to the enhanced responsivity.

Furthermore, since the polarity of the piezopotential relies on the relationship between direction of $c$-axis of ZnO NRs and applied strain, when applying tensile strain on ZnO NRs, a positive piezopotential will be created at the interface of the ZnO NRs and the electrolyte. To investigate this phenomenon, we changed the angles upward and measured the photocurrent response at zero bias with the UV light switching on and off, as shown in Fig. 4(a). As shown in Fig. 4(b), the responsivity decreased with the increasing of the upward angles. The SBH is plotted in Fig. 4(c), and it declined as the decreasing of the upward angles. We use the schematic of energy band structure to explain this phenomenon, as presented in Fig. 4(d). A positive piezopotential will be created at the interface under upward angles, which causes electrons to accumulate at the interface, resulting in a narrower depletion layer and a lower barrier height from $\Delta E_{0}$ to $\Delta E^{-}$. The decreased barrier height weakened the built in electric field. Thus, the recombination of the generated electron-hole pairs could be increased, resulting in the decreased responsivity. Electron-hole pairs could be increased, resulting in the decreased responsivity.

\section{Conclusions}

In summary, we successfully prepared the ZnO NRs on ITO coated PET substrate by a facile hydrothermal method. A flexible, stable, low-cost and self-powered UV photodetector is fabricated based on $\mathrm{ZnO}$ NRs and a novel iodine-free quasi solid-state electrolyte, which shows fast and high response to UV light at $0 \mathrm{~V}$. The rise and decay time are 0.03 and $0.13 \mathrm{~s}$, respectively. The responsivity is $50.5 \mathrm{~mA} \mathrm{~W}^{-1}$. The staininduced piezopotential on the performance of the ZnO NRs/ electrolyte was investigated in detail. About $63 \%$ enhanced responsivity could be achieved when the downward angles up to $60^{\circ}$. The enhancement mechanism is attributed to more favorable separation and transport of photogenerated electronhole pairs, which is a result of the modification effect of the negative piezopotential on the band structure at the interface between the ZnO NRs and the electrolyte. Moreover, the device exhibits good stability, and the performance has no obvious decay three months later. This work provides a guideline to further optimize the performance of a self-powered UV photodetector based on ZnO NRs and an iodine-free quasi solid-state electrolyte via strain engineering.

\section{Conflicts of interest}

There are no conflicts to declare.

\section{Acknowledgements}

This work was supported by National Natural Science Foundation of China under Grant No. 51302244 and 91333203, and Zhejiang Provincial Natural Science Foundation of China under Grant No. LY17E020005.

\section{References}

1 U. Ozgur, Y. I. Alivov, C. Liu, A. Teke, M. A. Reshchikov, S. Dogan, V. Avrutin, S. J. Cho and H. Morkoc, J. Appl. Phys., 2005, 98, 041301.

2 Z. L. Wang, J. Phys.: Condens. Matter, 2004, 16, R829-R858.

3 C. Klingshirn, ChemPhysChem, 2007, 8, 782-803.

4 A. B. Djurišić and Y. H. Leung, Small, 2006, 2, 944-961.

5 Y. K. Su, S. M. Peng, L. W. Ji, C. Z. Wu, W. B. Cheng and C. H. Liu, Langmuir, 2010, 26, 603-606.

6 A. B. Djurišić, A. M. C. Ng and X. Y. Chen, Prog. Quantum Electron., 2010, 34, 191-259.

7 S. M. Hatch, J. Briscoe and S. Dunn, Adv. Mater., 2013, 25, 867-871.

8 P. Lin, X. Chen, X. Yan, Z. Zhang, H. Yuan, P. Li, Y. Zhao and Y. Zhang, Nano Res., 2014, 7, 860-868.

9 S. I. Inamdar and K. Y. Rajpure, J. Alloys Compd., 2014, 595, $55-59$.

10 W. Dai, X. Pan, S. Chen, C. Chen, Z. Wen, H. Zhang and Z. Ye, J. Mater. Chem. C, 2014, 2, 4606.

11 Y. W. Shen, X. Q. Yan, H. N. Si, P. Lin, Y. C. Liu, Y. H. Sun and Y. Zhang, ACS Appl. Mater. Interfaces, 2016, 8, 6137-6143.

12 Z. Gao, W. Jin, Y. Zhou, Y. Dai, B. Yu, C. Liu, W. Xu, Y. Li, H. Peng, Z. Liu and L. Dai, Nanoscale, 2013, 5, 5576.

13 P. Ni, C. Shan, S. Wang, X. Liu and D. Shen, J. Mater. Chem. C, 2013, 1, 4445-4449.

14 A. Berendjchi, R. Khajavi, A. A. Yousefi and M. E. Yazdanshenas, J. Cleaner Prod., 2017, 149, 521-527.

15 W. Cho, Y. R. Kim, D. Song, H. W. Choi and Y. S. Kang, J. Mater. Chem. A, 2014, 2, 17746-17750.

16 N. Sharifi, F. Tajabadi and N. Taghavinia, ChemPhysChem, 2014, 15, 3902-3927.

17 X. Peng, Y. Zeng, X. Pan, W. Wang, Y. Zhou, F. Wang, Q. Lu and Z. Ye, $R S C A d v ., 2017,7,29440-29445$. 
18 S. Jeong, M. W. Kim, Y. Jo, Y. Leem, W. Hong, B. Kim and S. Park, Nano Energy, 2016, 30, 208-216.

19 X. Wang, J. Zhou, J. Song, J. Liu, N. Xu and Z. L. Wang, Nano Lett., 2006, 6, 2768-2772.

20 Z. L. Wang, Nano Today, 2010, 5, 540-552.

21 Z. L. Wang, J. Phys. Chem. Lett., 2010, 1, 1388-1393.

22 Z. L. Wang, Adv. Mater., 2012, 24, 4632-4646.

23 Y. Zhang, X. Yan, Y. Yang, Y. Huang, Q. Liao and J. Qi, Adv. Mater., 2012, 24, 4647-4655.

24 Y. Zeng, Z. Ye, B. Lu, W. Dai and X. Pan, Appl. Phys. A, 2016, 122, 296.

25 L. Vayssieres, Adv. Mater., 2003, 15, 464-466.

26 S. Xu and Z. L. Wang, Nano Res., 2011, 4, 1013-1098.

27 B. Zhao, F. Wang, H. Chen, L. Zheng, L. Su, D. Zhao and X. Fang, Adv. Funct. Mater., 2017, 27, 1700264.

28 B. Deka Boruah and A. Misra, ACS Appl. Mater. Interfaces, 2016, 8, 18182-18188.

29 H. Chen, K. Liu, X. Chen, Z. Zhang, M. Fan, M. Jiang, X. Xie, H. Zhao and D. Shen, J. Mater. Chem. C, 2014, 2, 9689-9694. 30 Q. Li, L. Wei, Y. Xie, K. Zhang, L. Liu, D. Zhu, J. Jiao, Y. Chen, S. Yan, G. Liu and L. Mei, Nanoscale Res. Lett., 2013, 8, 415.
31 O. Game, U. Singh, T. Kumari, A. Banpurkar and S. Ogale, Nanoscale, 2014, 6, 503-513.

32 W. Peng, X. Wang, R. Yu, Y. Dai, H. Zou, A. C. Wang, Y. He and Z. L. Wang, Adv. Mater., 2017, 29, 1606698.

33 Z. Wang, R. Yu, C. Pan, Z. Li, J. Yang, F. Yi and Z. L. Wang, Nat. Commun., 2015, 6, 8401.

34 J. Li, X. Wu, M. M. Shirolkar, M. Li, C. Xu and H. Wang, RSC Adv., 2017, 7, 18987-18992.

35 F. Yi, Q. Liao, Y. Huang, Y. Gu and Y. Zhang, Semicond. Sci. Technol., 2013, 28, 105023.

36 P. Lin, X. Yan, Y. Liu, P. Li, S. Lu and Y. Zhang, Phys. Chem. Chem. Phys., 2014, 16, 26697-26700.

37 S. Lu, J. Qi, S. Liu, Z. Zhang, Z. Wang, P. Lin, Q. Liao, Q. Liang and Y. Zhang, ACS Appl. Mater. Interfaces, 2014, 6, 1411614122.

38 P. Lin, X. Yan, Z. Zhang, Y. Shen, Y. Zhao, Z. Bai and Y. Zhang, ACS Appl. Mater. Interfaces, 2013, 5, 3671-3676.

39 R. Yang, Y. Qin, L. Dai and Z. L. Wang, Nat. Nanotechnol., 2009, 4, 34-39.

40 B. K. Sharma, N. Khare and S. Ahmad, Solid State Commun., 2009, 149, 771-774. 